\title{
Tailoring magnetic and dielectric properties of rubber ferrite composites containing mixed ferrites
}

\author{
M R ANANTHARAMAN*, K A MALINI, S SINDHU, E M MOHAMMED, S K DATE*, \\ S D KULKARNI ${ }^{*}, P$ A JOY ${ }^{\ddagger}$ and PHILIP KURIAN ${ }^{\dagger}$
}

Department of Physics, ${ }^{\dagger}$ Department of Polymer Science and Rubber Technology, Cochin University of Science and Technology, Cochin 682 022, India

†Physical Chemistry Division, National Chemical Laboratory, Pune 411 008, India

MS received 14 May 2001

\begin{abstract}
Rubber ferrite composites containing various mixed ferrites were prepared for different compositions and various loadings. The magnetic and dielectric properties of the fillers as well as the ferrite filled matrixes were evaluated separately. The results are correlated. Simple equations are proposed to predetermine the magnetic and dielectric properties. The validity of these equations is verified and they are found to be in good agreement. These equations are useful in tailoring the magnetic and dielectric properties of these composites with predetermined properties.
\end{abstract}

Keywords. Rubber ferrite composites; magnetic materials; magnetic composites; rubber magnets; mixed ferrites.

\section{Introduction}

Rubber ferrite composites (RFCs) are flexible elastomer magnets, in that they can be easily moulded into complex shapes. They find extensive applications as microwave absorbers and other devices, where flexibility and mouldability are important criteria. The microwave absorbing properties of these rubber ferrite composites warrant an appropriate magnetic permeability and a desired dielectric constant. The incorporation of these fillers modifies the magnetic property of the matrix and alters the dielectric properties (Naito and Mizumoto 1988; Mirtaheri et al 1989; Grunberger et al 1991; Safari Ardi et al 1995; Anantharaman et al 1996, 1999).

For applications, it is essential that the final properties are tailored by using simple relationship or equations so that RFCs with pre-determined properties can be prepared.

Ceramic fillers belonging to the series $\mathrm{Ni}_{1-x} \mathrm{Zn}_{x} \mathrm{Fe}_{2} \mathrm{O}_{4}$ (NZF) and $\mathrm{Mn}_{1-x} \mathrm{Zn}_{x} \mathrm{Fe}_{2} \mathrm{O}_{4}$ (MZF) for $x$ varying from 0 to 1 in steps of 0.2 were prepared by ceramic techniques and characterized. These pre-characterized fillers were then incorporated into two different matrixes viz. NZF in natural rubber (NR) and NZF in butyl rubber (BR) separately and MZF in natural rubber (NR) (Sung and Dae 1994; Anantharaman et al 1996; Malini et al 2001) according to a specific recipe. Prior to the incorporation of these fillers, the magnetic and dielectric

\footnotetext{
*Author for correspondence
}

properties of these fillers were evaluated. Further, after the incorporation, the magnetic and dielectric properties of the RFCs containing the respective fillers for various loadings of MZF in NR and NZF in BR and NR were also determined. The entire scheme of preparation of the filler, their incorporation and characterization are depicted in the form of a flow chart and is shown below.

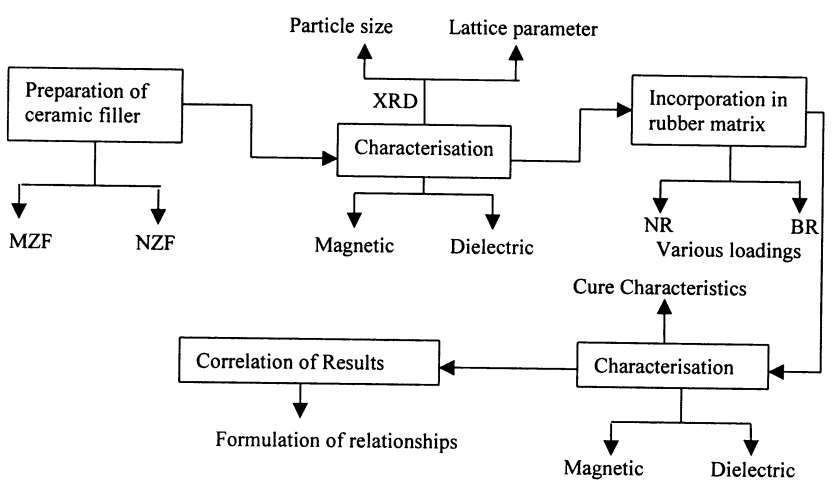

These results were then correlated based on the data generated. From the data thus generated a general equation which governs the saturation magnetization $\left(\sigma_{\mathrm{s}}\right)$ of fillers and RFCs is obtained and the validity of the equation is then verified with the observed values.

In a similar fashion, from the dielectric data generated on the ceramic fillers and RFCs, the validity of various forms of semi empirical relationships were tested by 
fitting the corresponding dielectric constant $(\varepsilon)$ of the components and then compared with the observed $\varepsilon$ of the composite. A most appropriate form of the relationship is finally proposed which is valid in general for all the samples and matrixes under consideration in this study.

The MZF and NZF series of mixed ferrites are chosen as the fillers since they are well studied ceramic systems and a wealth of information regarding various properties is available in the literature (Smit and Wijn 1959; Soshin 1964; Cullity 1972; Sung and Dae 1994; Malini et al 2001). Pre-characterized ferrite fillers were incorporated in the matrixes and care was taken to keep the conditions of preparations the same. Moreover, they can be prepared easily by conventional ceramic techniques (Raul 1994). NR and BR matrixes are selected since NR is an unsaturated matrix and available locally. They are easily mouldable. BR represents a saturated non-polar polymer with superior mechanical strength and is synthetic in nature. Besides, the properties also could be compared.

\section{Experimental}

\subsection{Preparation of $M Z F$ and NZF}

Freshly prepared FOD precursors were used to synthesize these fillers. AR grade oxides of $\mathrm{Mn}, \mathrm{Ni}$ and $\mathrm{Zn}$ were used as other constituent ingredients and they were prepared by conventional ceramic techniques (Raul 1994). The details are cited elsewhere (Anantharaman et al 1999; Malini et al 2001; Mohammed et al 2001a).

\subsection{Structural analysis}

The MZF and NZF powders were then analysed by X-ray powder diffraction techniques using Rigaku D-Max C $\mathrm{X}$-ray diffractometer. The identification of the phase and the evaluation of structural parameters were carried out (Cullity 1978; Anantharaman et al 1999; Malini et al 2001; Mohammed et al 2001a). For these, $\mathrm{CuK}_{\alpha}$ radiation with wavelength $\lambda=1.5405 \AA$ was employed.
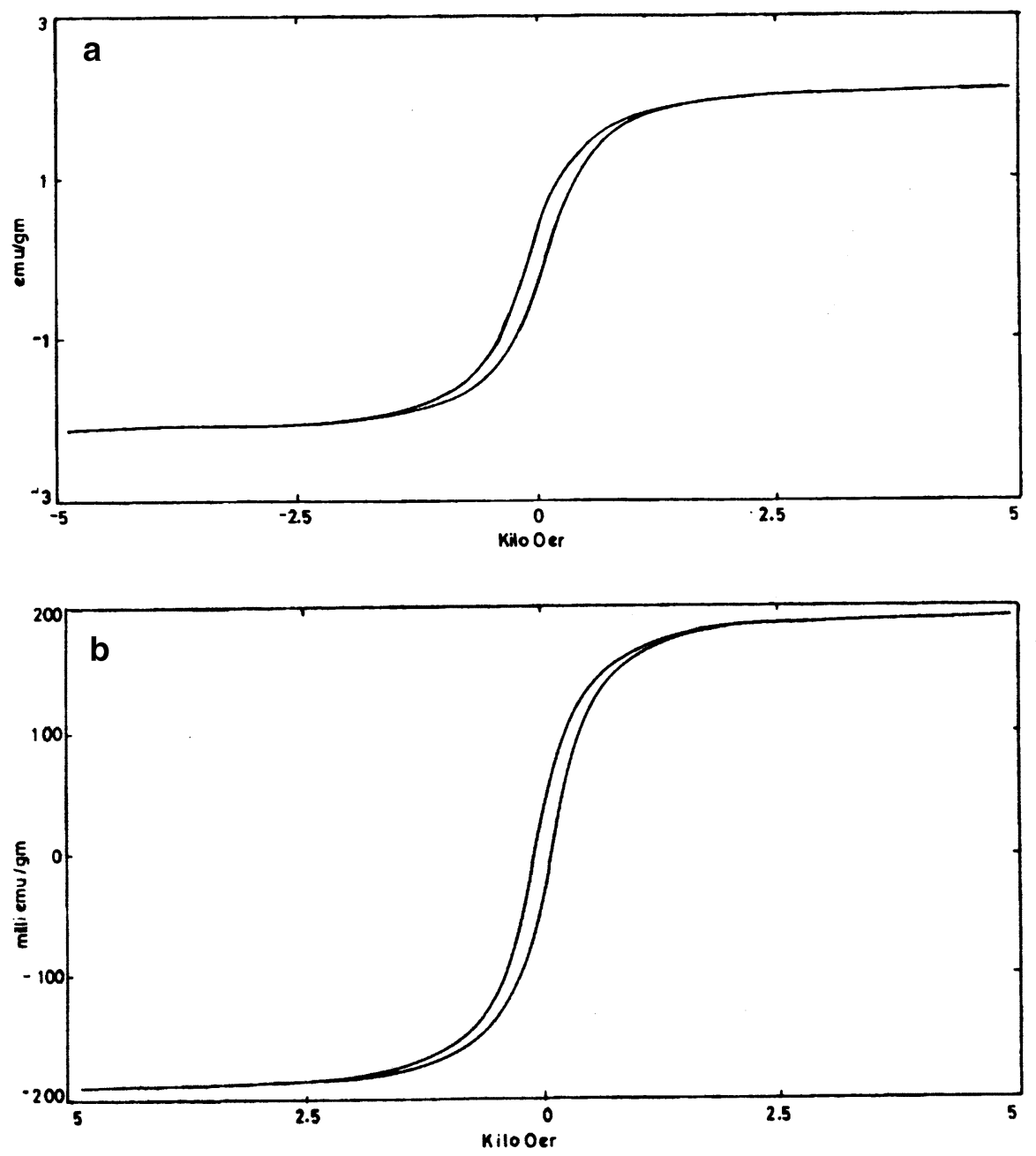

Figure 1. Representative hysteresis loop for a. ceramic NZF and b. RFC (NZF in NR). 


\subsection{Magnetic measurements}

Magnetic measurements were carried out by using a vibrating sample magnetometer (Model: 4500 from EG \& G PARC). Parameters such as saturation magnetization $\left(\sigma_{\mathrm{s}}\right)$ and coercivity $\left(H_{\mathrm{c}}\right)$ were obtained from these measurements.

\subsection{Dielectric measurements}

The dielectric constant and dielectric loss of these ferrite fillers and RFCs were determined using an LCR meter (model: HP 4285A) and a home made dielectric cell (Mohammed et al 2001b). The samples were made in the form of a pellet with a diameter of $12 \mathrm{~mm}$. The dielectric constant of the samples were evaluated using the relation

$$
C=\frac{\varepsilon_{0} \cdot \varepsilon_{\mathrm{r}} \cdot A}{d},
$$

where $A$ is the surface area of the sample, $d$ the thickness
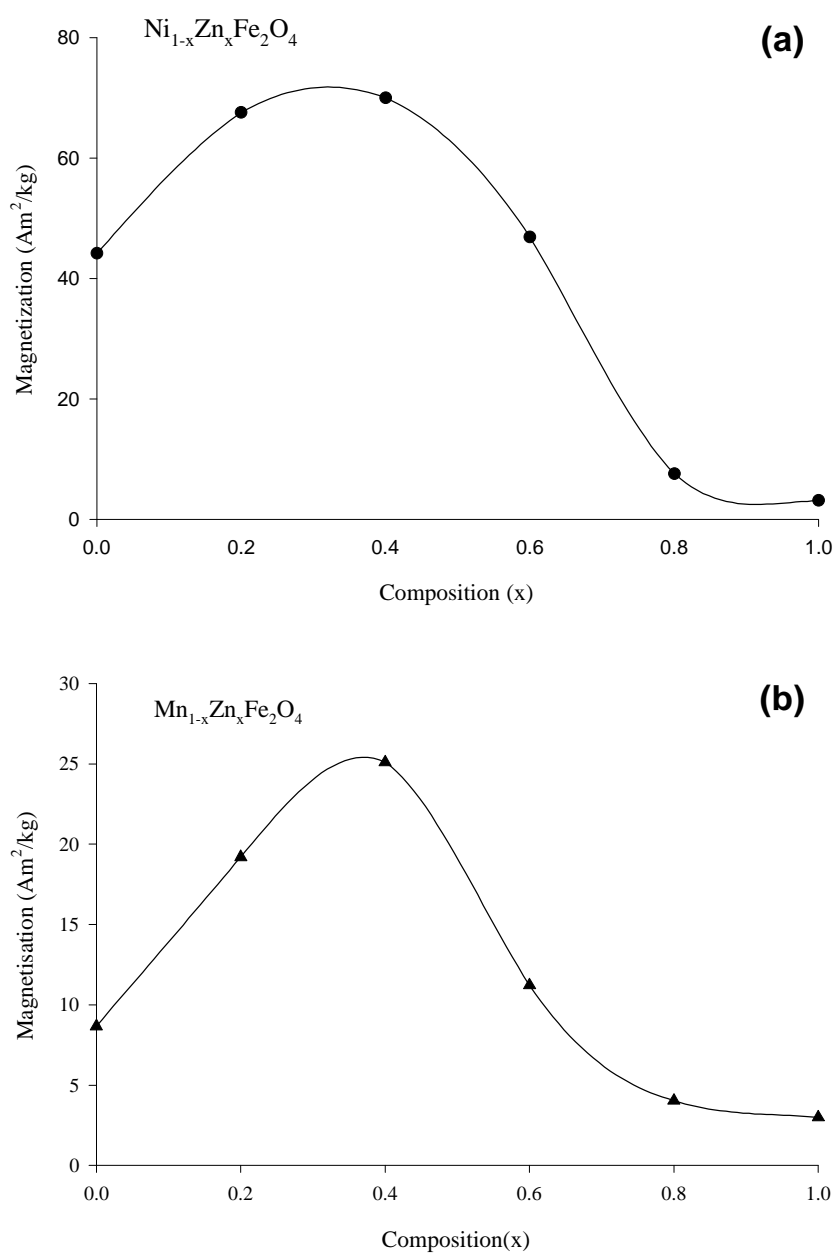

Figure 2. Variation of magnetization with composition for NZF and MZF. of the sample, $\varepsilon_{\mathrm{r}}$ the dielectric constant of the material, $\varepsilon_{0}$ the dielectric constant of air and $C$ the measured capacitance of the sample. The data acquisition as well as the calculation of $\varepsilon_{\mathrm{r}}$ and $\tan \delta$ for various frequencies and at different temperatures were fully automated using a package called Lab VIEW based on G-Programming. Appropriate modifications were incorporated in the software so as to enable the data acquisition automatic and visual observation of the graphs on the computer screen.
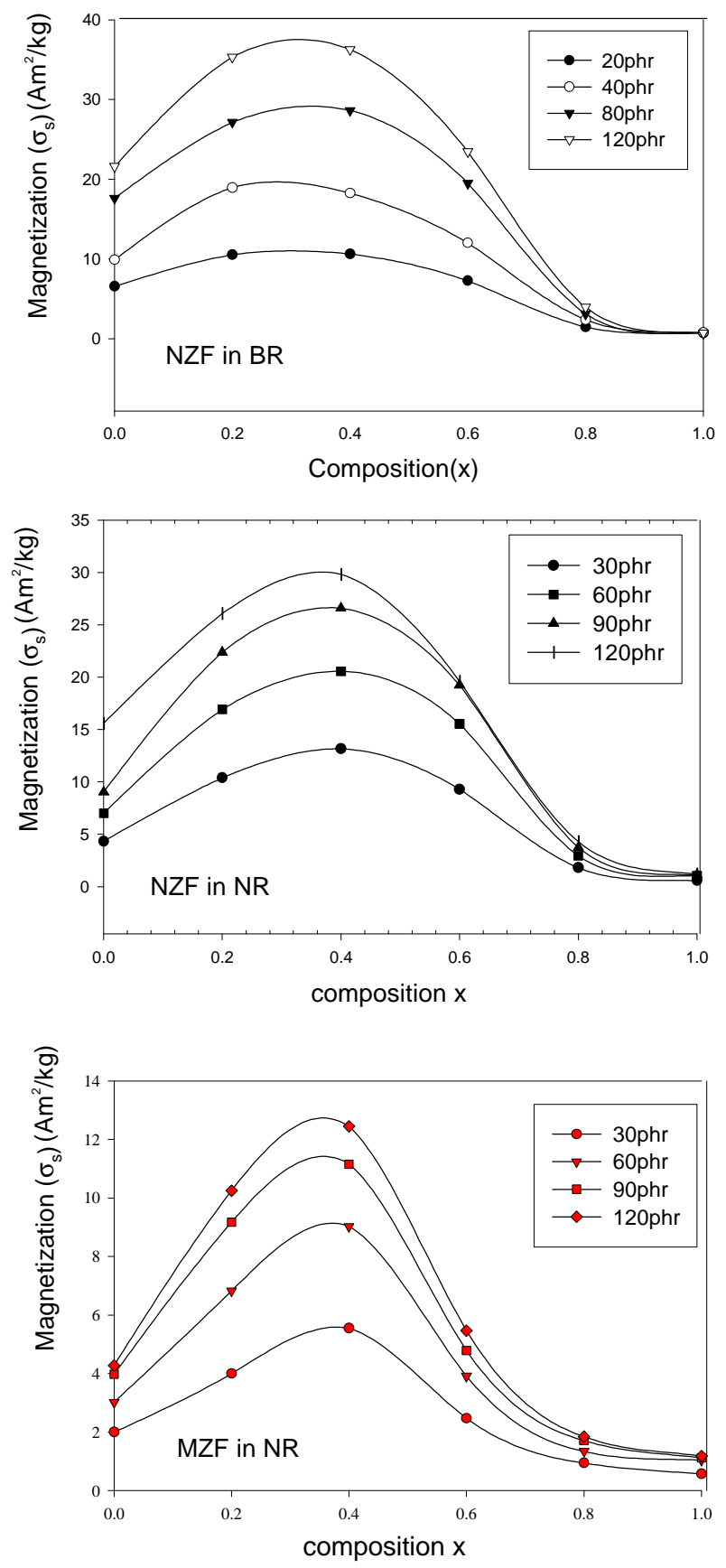

Figure 3. Variation of $\sigma_{\mathrm{s}}$ with composition for RFC for different loadings. 


\section{Results and discussion}

\subsection{Tailoring magnetic properties}

Parameters viz. saturation magnetization $\left(\sigma_{\mathrm{s}}\right)$, coercivity $\left(H_{\mathrm{c}}\right)$ and $\sigma_{\mathrm{r}} / \sigma_{\mathrm{s}}$ for various composition of the ceramic filler and for various loadings of RFCs, were evaluated from the VSM data. Representative hysteresis loop for ceramic and composites are shown in figures $1 \mathrm{a}$ and $\mathrm{b}$, respectively. Representative graphs for NZF and MZF for saturation magnetization $\left(\sigma_{\mathrm{s}}\right)$ for various $x$ is shown in
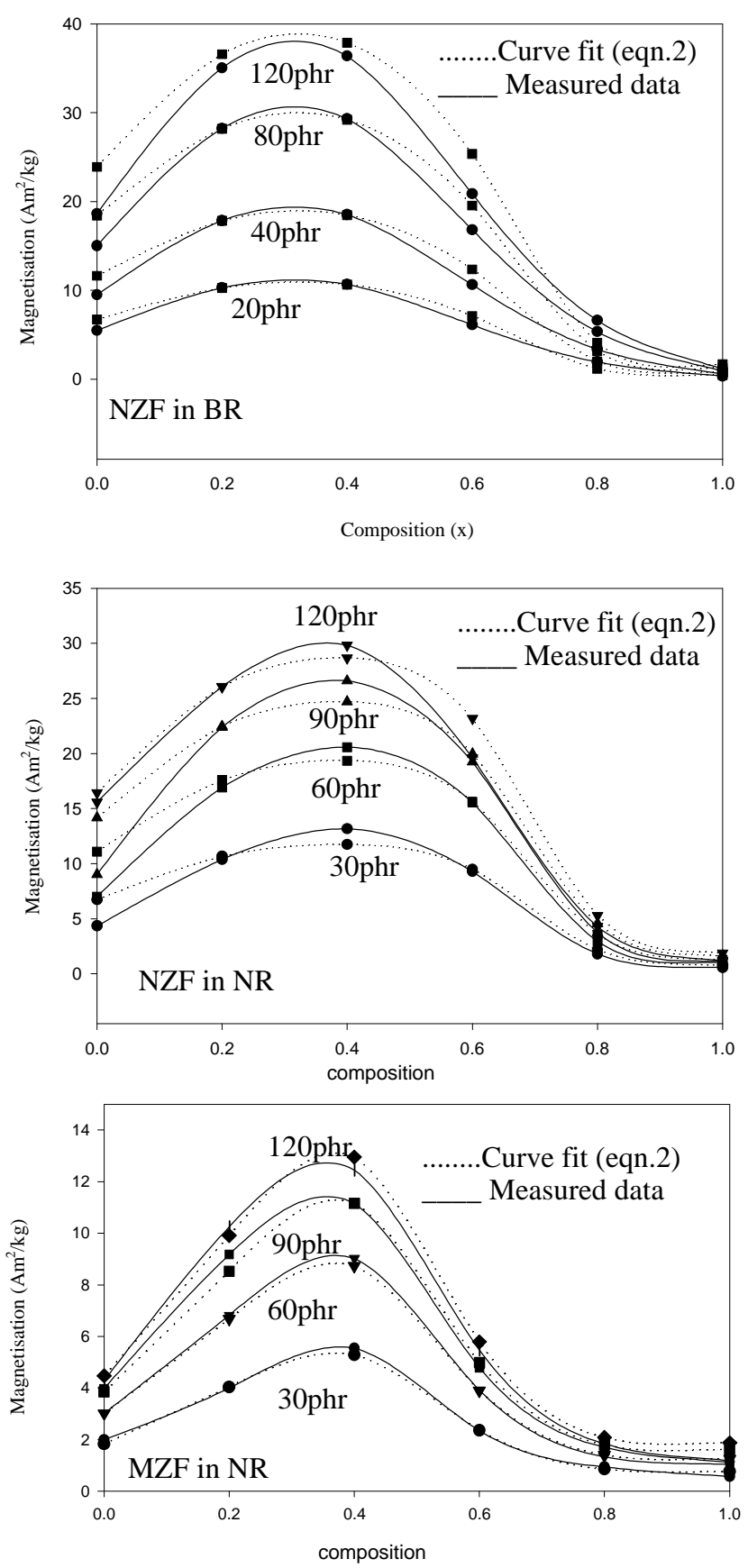

Figure 4. Measured and calculated values of magnetization (2). figure 2. Graphs for RFCs depicting the variation of $\sigma_{\mathrm{s}}$ with $x$ for various loadings are shown in figure 3 . Simple mixture equation of the general form

$$
\sigma_{\mathrm{rfc}}=W_{1} \sigma_{1}+W_{2} \sigma_{2},
$$

can be applied to evaluate the saturation magnetization of the composites. Here $W_{1}$ is the weight fraction of the filler, $\sigma_{1}$ the magnetization of the filler, $W_{2}$ the weight fraction of matrix and $\sigma_{2}$ the magnetization of the matrix.
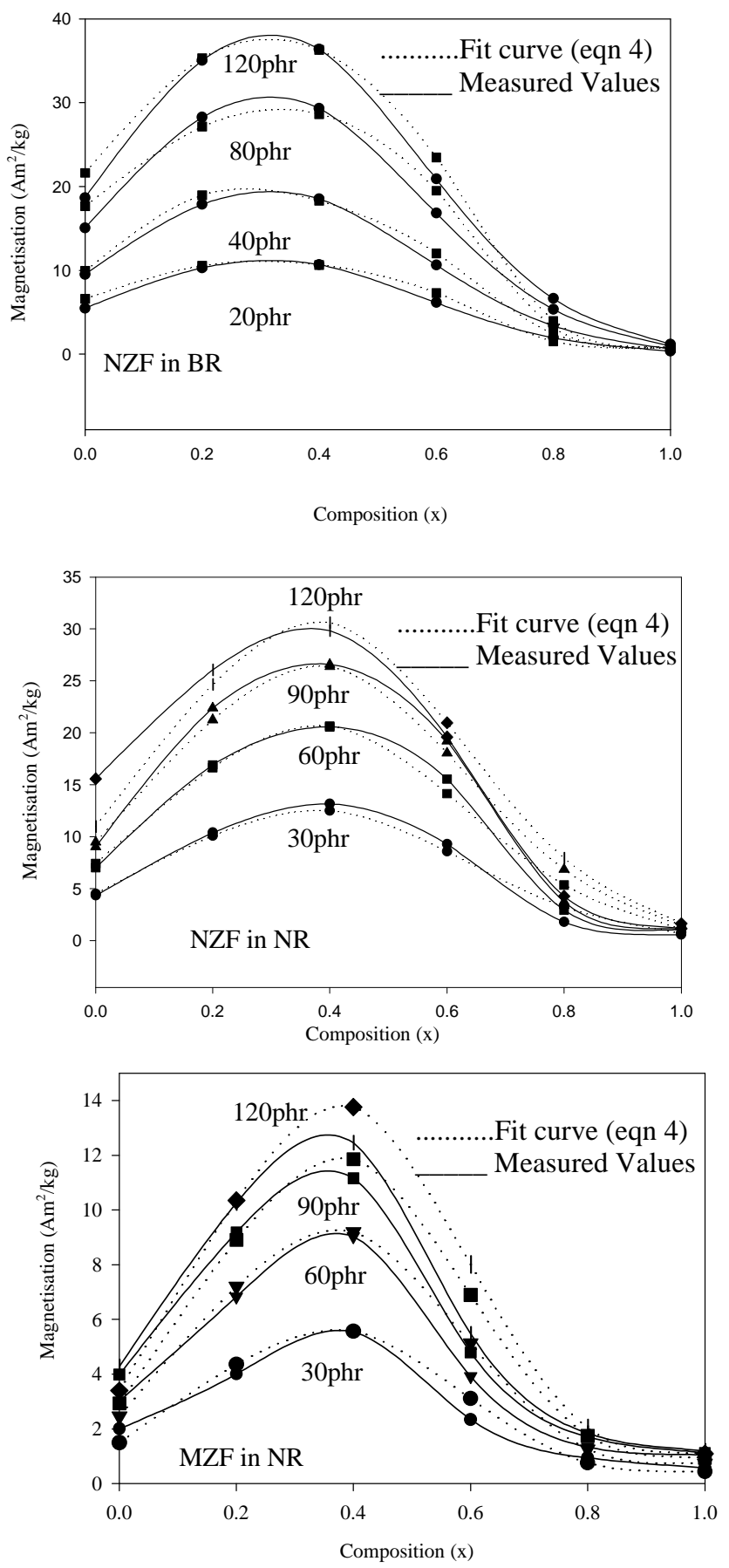

Figure 5. Measured and calculated values of magnetization (4). 
Since the matrix is nonmagnetic this equation is reduced to the form

$$
\sigma_{\mathrm{rfc}}=W_{1} \sigma_{1} \text {. }
$$

The $\sigma_{\mathrm{s}}$ values for composites containing MZF and NZF were estimated using (2) and the measured and calculated values of $\sigma_{\mathrm{s}}$ are shown in figure 4 . The measured and the calculated values using (2) are in agreement. This mixture equation requires the magnetization values $\left(\sigma_{\mathrm{s}}\right)$ corresponding to all $x$ values of the filler in order to pre-determine the magnetic properties.

An inspection of the magnetization $\left(\sigma_{\mathrm{s}}\right)$ vs composition $(x)$ for MZF in natural rubber (NR) and NZF in NR and BR (figure 3) indicate that they follow a Gaussian profile. These were then fitted into an equation of the following form

$$
\sigma_{\mathrm{rfc}}=A \cdot \exp \left[-0 \cdot 5\left(\frac{x-x_{0}}{b}\right)^{2}\right],
$$

where $A$ and $b$ are constants. Appropriate meanings were assigned to the coefficients from the observations on the
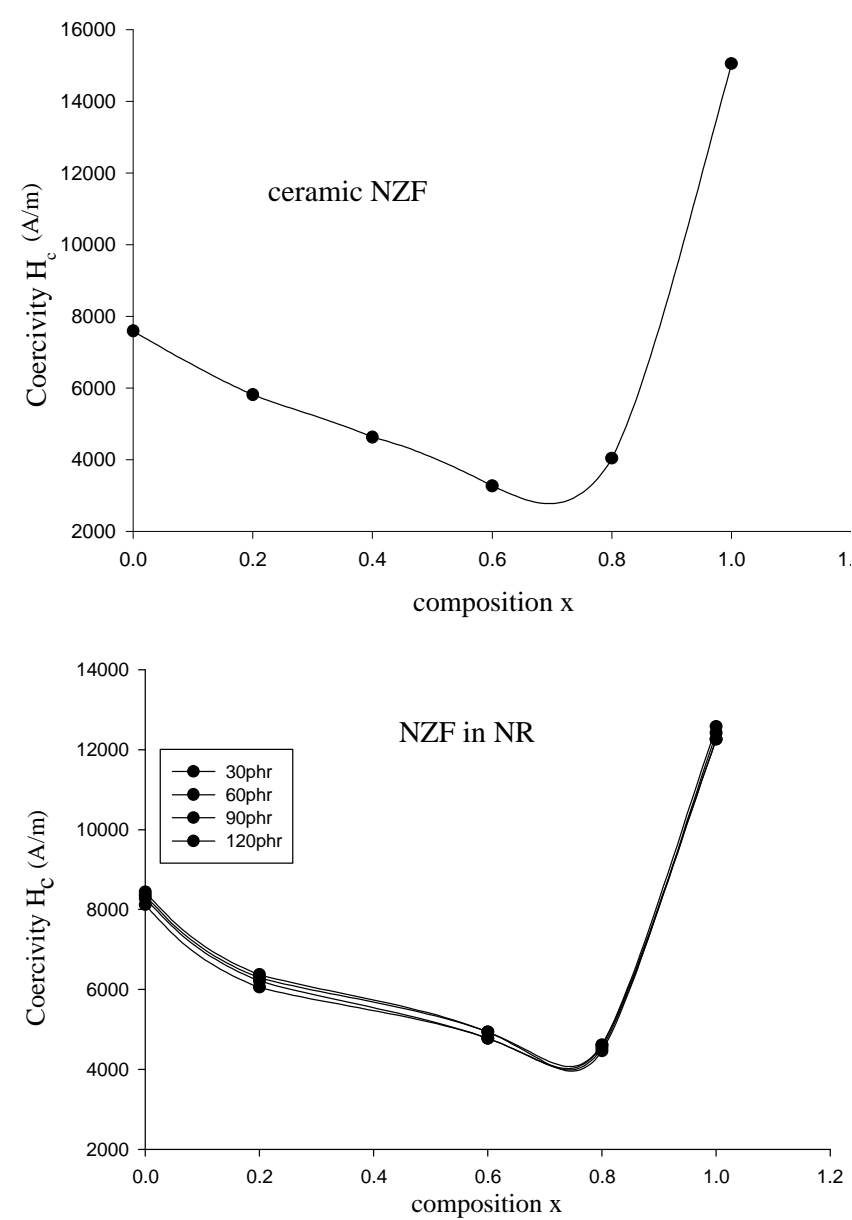

Figure 6. Variation of coercivity with composition for ceramic and RFC containing NZF. experimental value and a modified equation was formulated to relate the magnetization $\left(\sigma_{\mathrm{s}}\right)$ of RFCs and the magnetization $\left(\sigma_{\mathrm{s}}\right)$ of the ceramic fillers. The final fit after assigning appropriate meanings assume the following form

$$
\sigma_{\mathrm{rfc}}=(1+0 \cdot 2 x) \cdot \sigma_{\text {cer.max }} \cdot W_{1} \cdot \exp \left[-0 \cdot 5\left(\frac{x-x_{0}}{b}\right)^{2}\right],
$$

where $\sigma_{\text {cer.max }}$ and $x_{0}$ are the maximum saturation magnetization of the ceramic filler and its zinc content, respectively. The $\sigma_{\mathrm{rfc}}$ for MZF in NR and NZF in NR and BR were calculated with $b$ equal to $0 \cdot 26$. The calculated and observed values were compared and they are shown in figure 5. The observed and calculated values are in excellent agreement. It is to be noted that the modified equation requires only the magnetization, $\sigma_{\mathrm{s}}$ of that particular $x$ which shows maximum magnetization.

The evaluation of $H_{\mathrm{c}}$ for composites containing NZF and MZF shows that the $H_{\mathrm{c}}$ of RFCs equals the $H_{\mathrm{c}}$ of
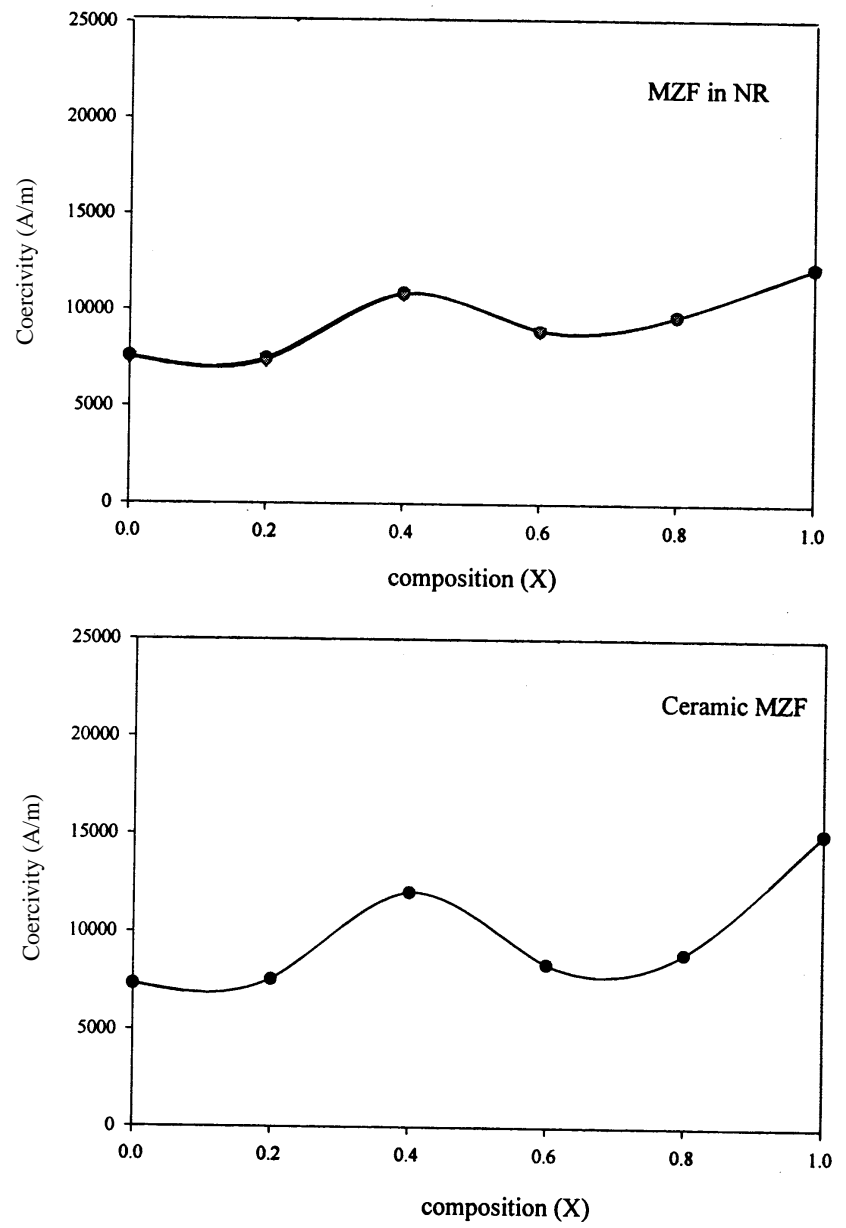

Figure 7. Variation of coercivity vs composition of MZF in NR and ceramic MZF. 
the respective ceramic component. Representative graphs showing the variation of $H_{\mathrm{c}}$ with composition for ceramic as well as composite samples for NZF and MZF are shown in figures 6 and 7, respectively. This means that RFCs can be tailor made with appropriate $\sigma_{\mathrm{s}}$ and $H_{\mathrm{c}}$ by a judicious choice of the filler belonging to a particular series. The weight fraction can be increased to acquire the required $\sigma_{s}$ by using (4). Care should be taken so as to not exceed the loading beyond the percolation threshold. In our set of experiments the percolation threshold was not yet reached for a loading of $120 \mathrm{phr}$ (parts per hundred rubber by weight). Equation (4) can be employed to pre-determine the magnetic properties of a composite containing a mixed ferrite filler exhibiting a Gaussian profile for $x$ vs $\sigma_{\mathrm{s}}$. As far as $\sigma_{\mathrm{s}}$ is concerned, in a mixed ferrite series showing a maximum magnetization at $x=0.4$ or $x=0.6$ can be selected and $\sigma_{\mathrm{s}}$ of the RFCs can be modulated by choosing the appropriate weight fraction. For applications where $H_{\mathrm{c}}$ is a determining factor, mixed ferrites containing cobalt or surface modified with $\mathrm{Co}$ is an excellent filler candidate for making RFCs with large coercivity. Appropriate heat-treated magnetic fillers also enhance the coercivity of fillers (Kaneko 1980; Anantharaman and Keer 1994).
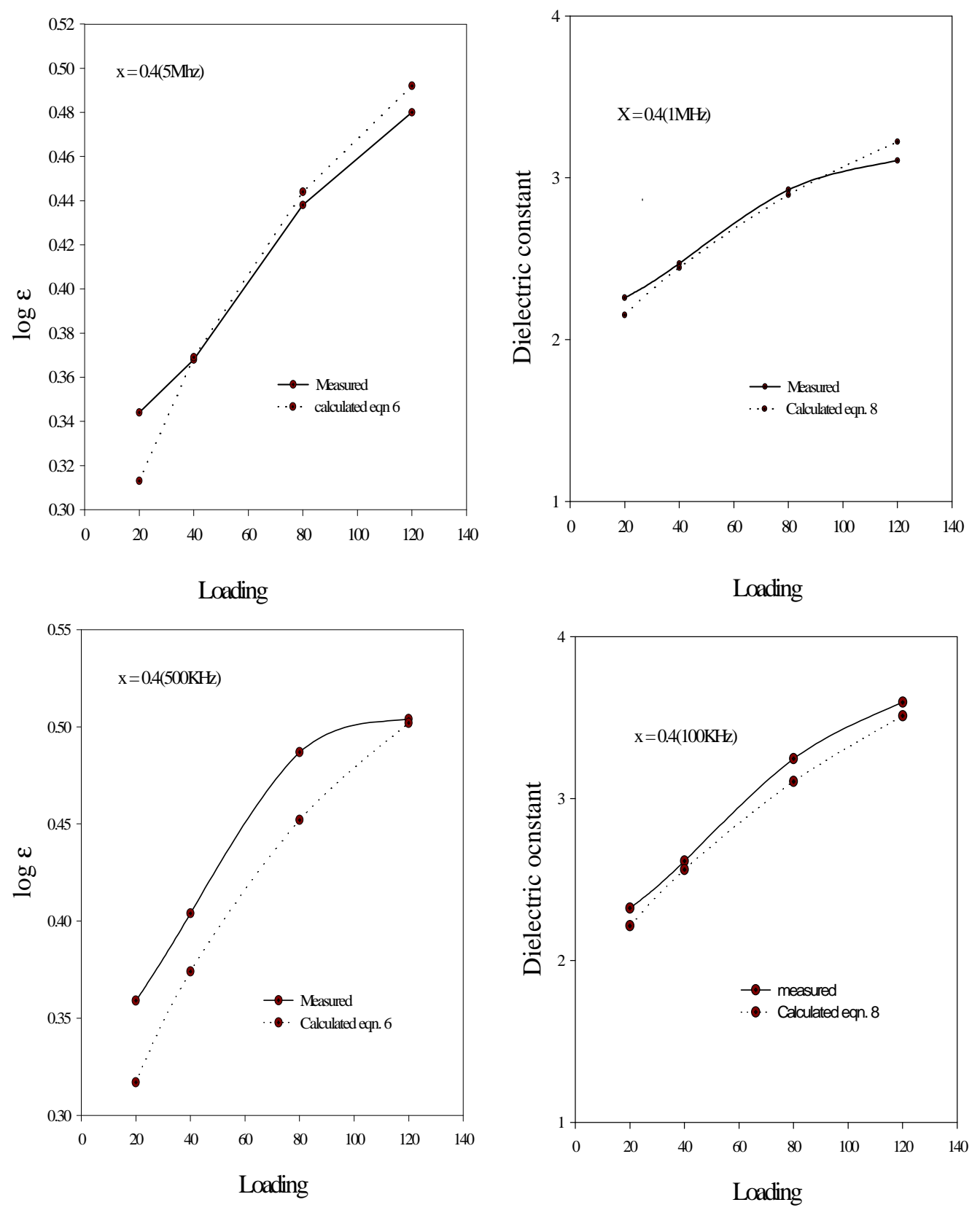

Figure 8. Calculated and measured value of dielectric constant for NZF in BR. 


\subsection{Tailoring dielectric properties}

The dielectric constants of the ceramic as well as composite samples of NZF and MZF in NR and BR were determined. The dielectric properties of blank matrix were also studied for different frequencies and for different temperatures. Attempts are also made to predict the dielectric properties of the composites from its constituents by considering some empirical relationships and formulae.

Composite dielectrics may be treated as chaotic or as a mixture of several components. Several semi empirical relationships and formulae are suggested by various researchers for the evaluation of permittivity of composites from the permittivity of the constituents of the composite (Musal et al 1988; Ki et al 1995). These are based on various theoretical presumptions. For example, for a mixture of $m$ components the dielectric constant $\varepsilon^{*}$ is connected by a relation

$$
\log \varepsilon^{*}=\sum_{i=1}^{m} y_{i} \cdot \log \varepsilon_{i},
$$

where $\varepsilon^{*}$ is the dielectric constant of the mixture and $y$ the volume fraction of the component. For a two-component
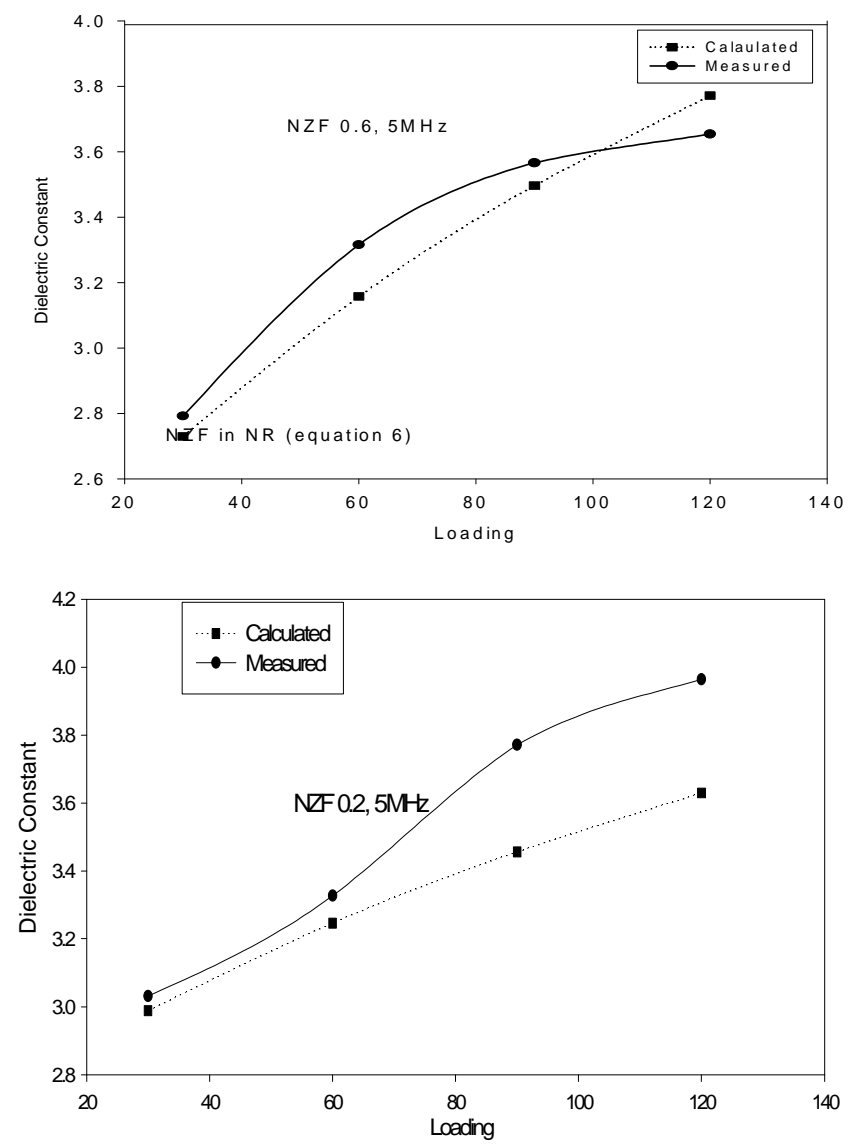

Figure 9. Measured and calculated values of dielectric constant for NZF in NR. system the relationship can be written as

$$
\log \varepsilon^{*}=y_{1} \log \varepsilon_{1}+y_{2} \log \varepsilon_{2}
$$

where $\varepsilon^{*}$ is the dielectric constant of the composite, $\varepsilon_{1}, y_{1}$ and $\varepsilon_{2}, y_{2}$ are the dielectric constant and volume fractions of the matrix and the filler component, respectively.

Another mixture equation

$$
\varepsilon^{*}=\frac{\varepsilon_{1} \varepsilon_{2}}{\varepsilon_{1} y_{2}+\varepsilon_{2} y_{1}}
$$

is also found to be useful for predicting the loading dependence of dielectric constant of the RFC.

For a two-component system consisting of a non-polar matrix and insulating filler of the ferrite type the relationship can be written with the help of an equation of the form

$$
\varepsilon^{*}=\varepsilon_{1} \cdot\left\{\frac{\left[2 \varepsilon_{1}+\varepsilon_{2}+2 y\left(\varepsilon_{2}-\varepsilon_{1}\right)\right]}{\left[2 \varepsilon_{1}+\varepsilon_{2}-y\left(\varepsilon_{2}-\varepsilon_{1}\right)\right]}\right\},
$$

where $\varepsilon_{1}$ is the dielectric constant of the blank matrix, $\varepsilon_{2}$ the dielectric constant of the uniformly distributed (by volume) spherical inclusions and let $y$ be the volume fraction of the inclusion and $\varepsilon^{*}$ the dielectric constant of the matrix mixture or the composite.

Equations (6) and (8) were used to evaluate the permittivity of the composite (RFCs (matrix butyl rubber) containing $\mathrm{Ni}_{1-x} \mathrm{Zn}_{x} \mathrm{Fe}_{2} \mathrm{O}_{4}$ ) from the measured values of $\varepsilon_{1}$ and $\varepsilon_{2}$ which are the permittivities of matrix and filler, respectively. The observed values and the calculated values using (6) and (8) are shown in figure 8. From the

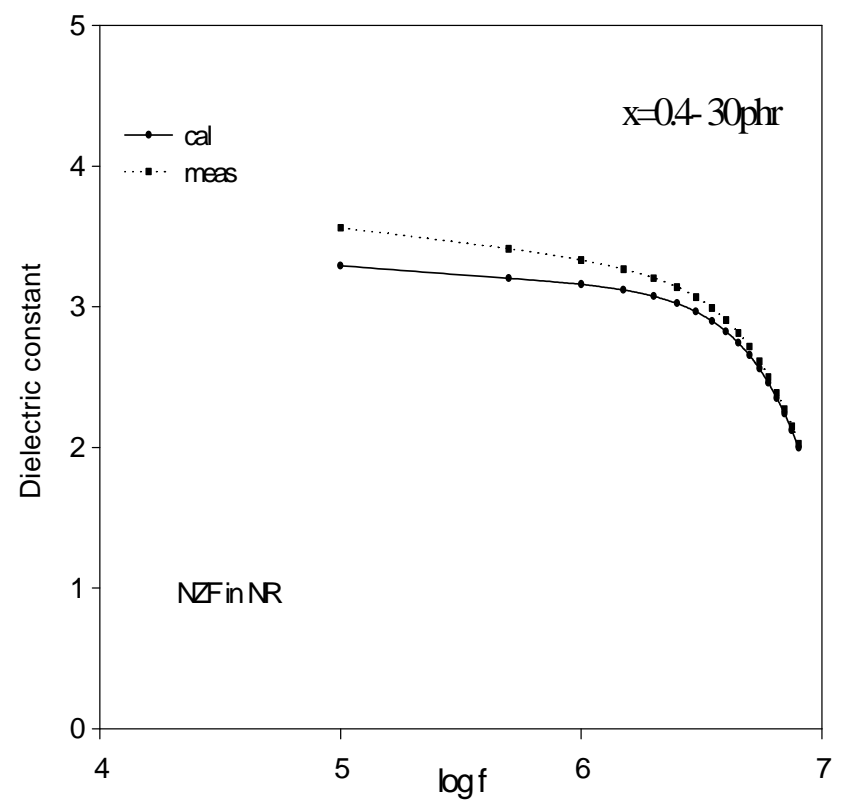

Figure 10. Representative graph showing the validity of (6) for all frequencies. 

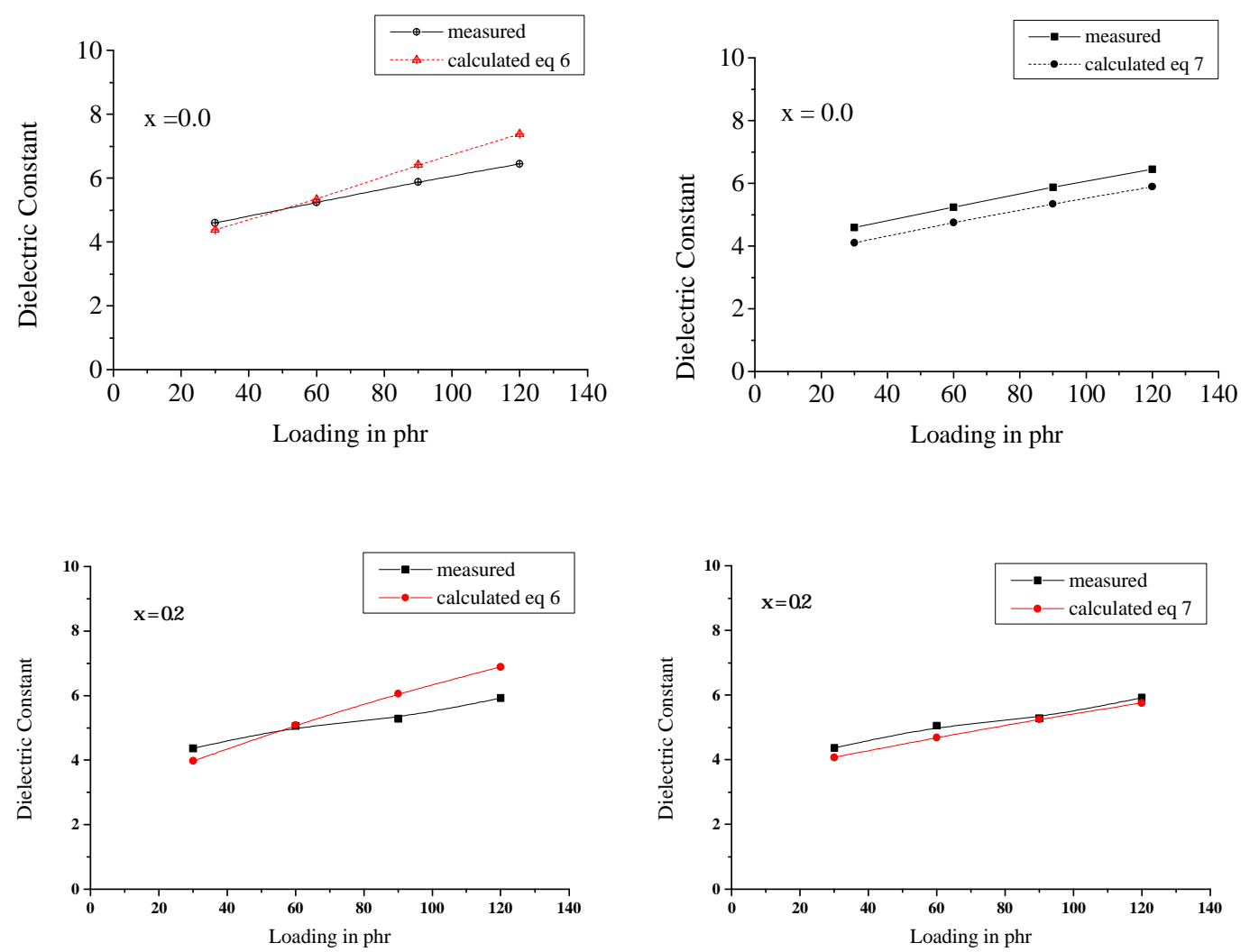

Figure 11. Representative graphs showing the validity of (6) and (7) for different compositions of MZF in NR.

figure it can be seen that they are in good agreement for almost all frequencies $(100 \mathrm{kHz}-5 \mathrm{MHz})$ and for various loadings (up to $120 \mathrm{phr}$ ).

Equations (6) and (7) were also tried for the evaluation of permittivity of RFCs containing NZF in NR matrix and they are shown in figure 9 . From the graphs it can be seen that the calculated values are in excellent agreement with the observed values. A representative graph to show the validity of (6) for the entire frequency range is given in figure 10 .

An excellent agreement was obtained for measured and calculated values of the dielectric constant using (6) for a system consisting of $\mathrm{Mn}_{1-x} \mathrm{Zn}_{x} \mathrm{Fe}_{2} \mathrm{O}_{4}$ in natural rubber matrix. The results are depicted in figure 11 .

Another equation of the form

$$
\varepsilon^{*}=\frac{\varepsilon_{1}}{(1-y)^{3}}
$$

is also available for the evaluation of the permittivity of the composites from the individual permittivity of the component in the composite. Here $\varepsilon_{1}$ is the permittivity of the matrix and $y$ the volume fraction of the inclusion or filler. Attempts were made to calculate the dielectric constant using this relation. But it was observed that this formula is useful for a composite containing conducting filler and cannot be applied to a composite containing an insulating filler.
From these observations it may be noted that (6) seems to be producing a better fit for all frequencies and for all loadings. Thus it would be appropriate to conclude that (6) can be used for the evaluation of permittivity of any system for all loadings and for all frequencies under consideration in this set of experiments.

\section{Conclusions}

As far as the prediction of $\sigma_{\mathrm{s}}$ of RFCs are concerned the general equation of the form

$$
\sigma_{\mathrm{rfc}}=(1+0 \cdot 2 x) \cdot \sigma_{\text {cer.max }} \cdot W_{2} \cdot \exp \left[-0 \cdot 5\left(\frac{x-x_{0}}{b}\right)^{2}\right],
$$

where $b \approx 0.26$ is suitable. It is to be noted that the calculation requires only the maximum magnetization value corresponding to the composition $x_{0}$. Calculations and comparisons have shown that this fits for all the studied system in the present investigation and it is to be assumed that a general equation of the above form can be employed to pre-determine the $\sigma_{\mathrm{s}}$ of RFCs containing any mixed ferrite series. A number of empirical relationships and formulae were tested for both NZF and MZF system to predict the dielectric property of the composite from its constituents. A general logarithmic equation of the form 


$$
\log \varepsilon^{*}=y_{1} \log \varepsilon_{1}+y_{2} \log \varepsilon_{2},
$$

is most appropriate for all the systems in the present study.

\section{Acknowledgements}

One of the authors (MRA) thanks the All India Council for Technical Education (AICTE) for financial assistance received in the form of a project under TAPTEC, GOI (F.No. 8017/RDII/MAT/30/98 dated 6 March 1998). Also, (KAM) thanks the Council of Scientific and Industrial Research, New Delhi, for a Research Fellowship and (EMM) thanks the University Grants Commission, New Delhi for a fellowship. (SS) thanks DST for a fellowship.

\section{References}

Anantharaman M R and Keer H V 1994 Magnetism: Rocks to superconductors, memoir 29 (Bangalore: Geological Society of India) pp 243-251

Anantharaman M R, Kurian P, Banerjee B, Mohammed E M and George M 1996 Kautschuk Gummi Kunststoffe 49424

Anantharaman M R, Sindhu S, Jagatheesan S, Malini K A and Philip Kurian 1999 J. Phys. D Appl. Phys. 321801

Cullity B D 1972 Introduction to magnetic materials (Philippines: Addison Wesley Publishing Co.)
Cullity B D 1978 Elements of X-ray diffraction (USA: Addison Wesley Pub. Co. Inc.) 2nd ed.

Grunberger A, Springmann B, Brusberg M, Schmidt M and Jahnke R 1991 J. Magn. Magn. Mater. 101173

Kaneko M 1980 IEEE Trans. Magn. MAG-16

Ki Chulhan, Hyung Do Choi, Tak Jin Moon, Wang Sup Kim and Kyung Yong Kim 1995 J. Mater. Sci. 303567

Malini K A, Mohammed E M, Sindhu S, Joy P A, Date S K, Kulkarni S D, Kurian P and Anantharaman M R 2001 J. Mater. Sci. (in press)

Mirtaheri S A, Yin J, Seki H, Mizumoto T and Naito Y 1989 Trans. Inst. Electron. Commun. Engg. Jpn E72 1447

Mohammed E M, Malini K A, Joy P A, Date S K, Kulkarni S D, Kurian P and Anantharaman M R 2001a Mater. Res. Bull. (submitted)

Mohammed E M et al 2001b Plastics, Rubber and Composites - Processing \& Applications (submitted)

Musal Jr H M, Hahn H T and Bush G G 1988 J. Appl. Phys. 63 3768

Naito Y and Mizumoto T 1988 Electr. Commun. Jpn 71

Raul Valenzuela 1994 Magnetic ceramics (Great Britain: Cambridge University Press)

Safari Ardi M, Dick W and McQueen D H 1995 Plastics, Rubber and Composites - Processing and Applications 24157

Soshin Chikazumi 1964 Physics of magnetism (New York: John Wiley \& Sons Inc.)

Smit J and Wijn H P G 1959 Ferrites (The Netherlands: Phillips Technical Library)

Sung Soo Kim and Dae Hee Han 1994 IEEE Trans. Mag. 30 4554 